\title{
Strong Internal and External Luminescence as Solar Cells Approach the Shockley-Queisser Limit
}

\author{
Owen D. Miller, Eli Yablonovitch, and Sarah R. Kurtz
}

\begin{abstract}
Absorbed sunlight in a solar cell produces electrons and holes. However, at the open-circuit condition, the carriers have no place to go. They build up in density, and ideally, they emit external luminescence that exactly balances the incoming sunlight. Any additional nonradiative recombination impairs the carrier density buildup, limiting the open-circuit voltage. At open circuit, efficient external luminescence is an indicator of low internal optical losses. Thus, efficient external luminescence is, counterintuitively, a necessity for approaching the Shockley-Queisser (SQ) efficiency limit. A great solar cell also needs to be a great light-emitting diode. $\mathrm{Ow}$ ing to the narrow escape cone for light, efficient external emission requires repeated attempts and demands an internal luminescence efficiency $\gg 90 \%$.
\end{abstract}

Index Terms-External luminescence, GaAs, Shockley-Queisser (SQ) limit, solar cells.

\section{INTRODUCTION}

$\mathbf{T}$ HE Shockley-Queisser (SQ) efficiency limit [1] for a single-junction solar cell is $\sim 33.5 \%$ under the standard AM1.5G flat-plate solar spectrum [2]. In fact, detailed calculations in this paper show that GaAs is capable of achieving this efficiency. Nonetheless, the record GaAs solar cell had achieved only $26.4 \%$ efficiency [3] in 2010. Previously, the record had been $26.1 \%$ [4] and prior to that stuck [5] at $25.1 \%$, during 1990-2007. Why then is there a 7\% discrepancy between the theoretical limit $33.5 \%$ versus the previously achieved efficiency of $26.4 \%$ ?

It is usual to blame material quality, but in the case of GaAs double heterostructures, the material is almost ideal [6] with an internal luminescence yield of $>99 \%$. This deepens the puzzle as to why the full theoretical SQ efficiency has yet to be achieved.

\section{Physics ReQuired to APPROACH THE SHOCKLEY-QUEISSER LIMIT}

Solar cell materials are often evaluated on the basis of two properties: how strongly they absorb light and whether the

Manuscript received July 21, 2011; revised February 9, 2012 and April 5, 2012; accepted April 16, 2012. Date of current version June 18, 2012. This work was supported by the U.S. Department of Energy "Light-Material Interactions in Energy Conversion" Energy Frontier Research Center under Grant DE-SC0001293. The work of O. D. Miller was supported by the National Science Foundation's Fellowship.

O. D. Miller and E. Yablonovitch are with the Material Sciences Division, Lawrence Berkeley National Laboratory, Berkeley, CA 94720 USA, and also with the Department of Electrical Engineering and Computer Sciences, University of California, Berkeley, CA 94720 USA (e-mail: omiller@ berkeley.edu; eliy@eecs.berkeley.edu).

S. R. Kurtz is with the National Renewable Energy Laboratory, Golden, CO 80401 USA (e-mail: sarah_kurtz@nrel.gov).

Color versions of one or more of the figures in this paper are available online at http://ieeexplore.ieee.org.

Digital Object Identifier 10.1109/JPHOTOV.2012.2198434 created charge carriers reach the electrical contacts successfully. Indeed, the short-circuit current in the solar cell is determined entirely by those two factors. However, the power output of the cell is determined by the product of the current and voltage, and it is, therefore, imperative to understand what material properties (and solar cell geometries) produce high voltages. We show here that maximizing the external emission of photons from the front surface of the solar cell proves to be the key to reaching the highest possible voltages. In the search for optimal solar cell candidates, materials that are good radiators, in addition to being good absorbers, are most likely to reach high efficiencies.

As solar efficiency begins to approach the SQ limit, the internal physics of a solar cell transforms, such that photonic considerations overtake electronic ones. Shockley and Queisser showed that high solar cell efficiency is accompanied by a high concentration of carriers, and by strong luminescent emission of photons. In a good solar cell, the photons that are emitted internally are likely to be trapped, reabsorbed, and reemitted at open circuit.

The SQ limit assumes perfect external luminescence yield at open circuit. On the other hand, inefficient external luminescence at open circuit is an indicator of nonradiative recombination and optical losses. Owing to the narrow escape cone, efficient external emission requires repeated escape attempts and demands an internal luminescence efficiency $\gg 90 \%$. We find that the failure to efficiently extract the recycled internal photons is an indicator of an accumulation of nonradiative losses, which are largely responsible for the failure to achieve the SQ limit in the best solar cells.

In high-efficiency solar cells, it is important to engineer the photon dynamics. The SQ limit requires $100 \%$ external luminescence to balance the incoming sunlight at open circuit. Indeed, the external luminescence is a thermodynamic measure of the available open-circuit voltage [7]. Owing to the narrow escape cone for internal photons, they find it hard to escape through the semiconductor surface. Except for the limiting case of a perfect material, external luminescence efficiency is always significantly lower than internal luminescence efficiency. Then, the SQ limit is not achieved.

The extraction and escape of internal photons is now recognized as one of the most pressing problems in light-emitting diodes (LEDs). In this paper, we assert that luminescence extraction is equally important to solar cells. The SQ limit cannot be achieved unless light extraction physics is designed into highperformance solar cells, just as in LEDs.

In some way, this is counterintuitive, since an extracted photon cannot contribute to performance. Paradoxically, $100 \%$ external extraction at open circuit is exactly what is needed to achieve the SQ limit. The paradox is resolved by recognizing 


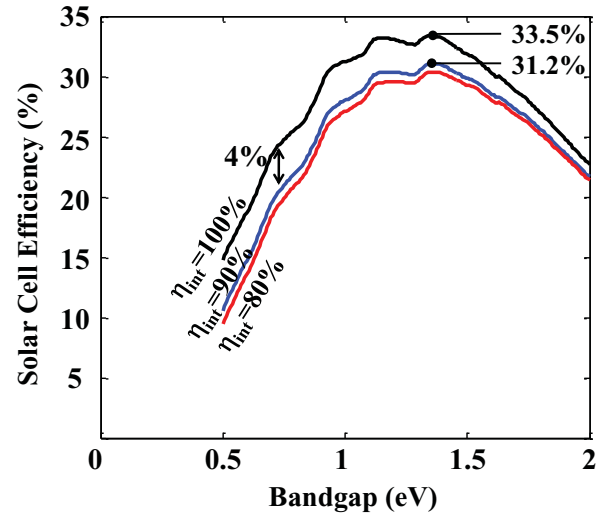

Fig. 1. Drastic effect of internal luminescence efficiency $\eta_{\text {int }}$ on theoretical solar cell efficiency. The shortfall is particularly noticeable for smaller bandgaps. A reduction from $\eta_{\text {int }}=100 \%$ to $\eta_{\text {int }}=90 \%$ already causes a large drop in performance, while a reduction from $\eta_{\text {int }}=90 \%$ to $\eta_{\text {int }}=80 \%$ causes little additional damage. Owing to the need for photon recycling and the multiple attempts required to escape the solar cell, $\eta_{\text {int }}$ must be $\gg 90 \%$.

that high extraction efficiency at open circuit is an indicator, or a gauge, of small optical losses. Previous record solar cells have typically taken no account of light extraction, resulting in the poor radiative efficiencies calculated in [8]. Nonetheless, approaching the $33.5 \%$ SQ limit will require light extraction to become part of all future designs. The present shortfall below the SQ limit can be overcome.

A recent paper by Green [8] reinforces the importance of light extraction. The record solar cells that have reached the highest efficiencies are also the ones with the highest external luminescence yield.

Although Silicon makes an excellent solar cell [9], Auger recombination fundamentally limits its internal luminescence yield to $<20 \%$ [10]-[12], which prevents Silicon from approaching the SQ limit. The physical issues presented here pertain to any material that has the possibility of approaching the SQ limit, which requires near unity external luminescence as III-V materials can provide, and that perhaps other material systems can provide as well.

Since light is trapped by total internal reflection, it is likely to be reabsorbed, leading to a further reemission event. With each absorption/reemission event, the solid angle of the escape cone [13] allows only $\left(1 / 4 n^{2}\right) \sim 2 \%$ of the internal light to escape. As a result, 1-sun incident can produce an internal photon density equivalent to up to 50 sun. This puts a very heavy burden on the parasitic losses in the cell. With only $2 \%$ escaping per emission event, even a $90 \%$ internal luminescence yield on each cycle would appear inadequate. Likewise, the rear mirror should have $\gg 90 \%$ reflectivity. This is illustrated in Figs. 1 and 2.

A good solar cell should be designed as a good LED, with good light extraction. In a way, this is not surprising. Most ideal machines work by reciprocity, that is, equally well in reverse. This has important ramifications. For ideal materials, the burden of high open-circuit voltage, and thereby high efficiency, lies with optical design: The solar cell must be designed for optimal light extraction under open-circuit conditions.
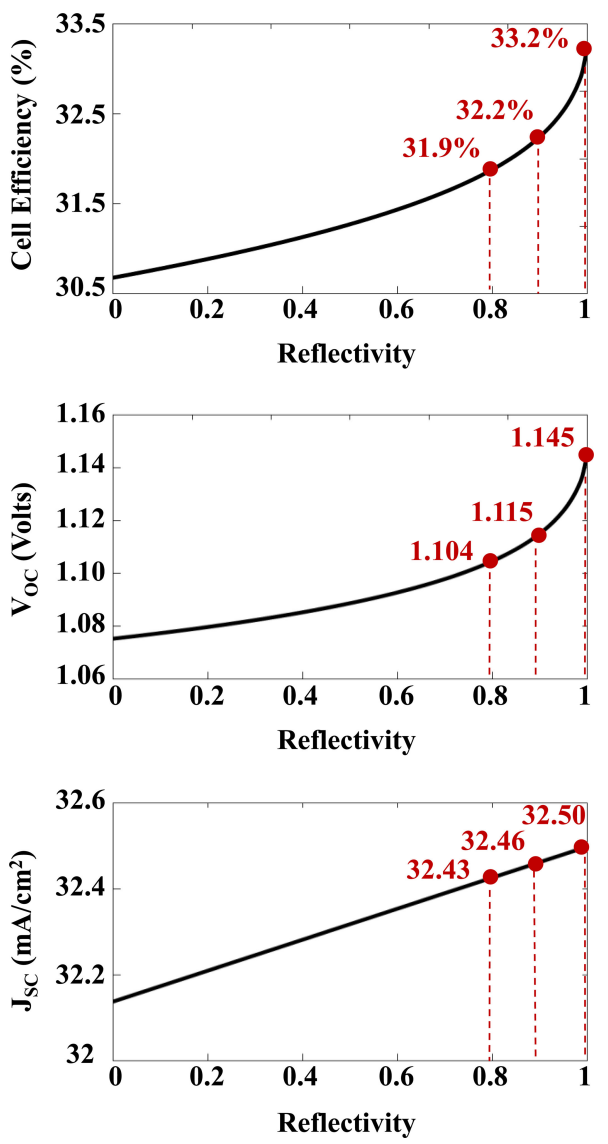

Fig. 2. Drastic effect of rear mirror reflectivity on cell efficiency and on opencircuit voltage $V_{\mathrm{OC}}$ but not on short-circuit current $J_{\mathrm{SC}}$ for a $3-\mu$ m-thick GaAs solar cell. Mirror reflectivity $\gg 90 \%$ makes a big difference, owing to the small escape cone for external emission and the multiple attempts needed for escape.

The assumption of perfect internal luminescence yield is a seductive one. The SQ limit gets a significant boost from the perfect photon recycling that occurs in an ideal system. Unfortunately, for most materials, their relatively low internal luminescence yields mean that the upper bounds on their efficiencies are much lower than the SQ limit. For the few material systems that are nearly ideal, such as GaAs, there is still a tremendous burden on the optical design of the solar cell. A very good rear mirror, for example, is of the utmost importance. In addition, it becomes clear that realistic material radiative efficiencies must be included in a credible assessment of any materials' prospects as a solar cell technology.

There is a well-known detailed balance equation relating the spontaneous emission rate of a semiconductor to its absorption coefficient [14]. Nevertheless, it is not true that all good absorbers are good emitters. If the nonradiative recombination rate is higher than the radiative rate, then the probability of emission will be very low. Amorphous silicon, for example, has a very large absorption coefficient of about $10^{5} / \mathrm{cm}$, yet the probability of emission at open circuit is $\sim 10^{-4}$ [8]. The probability of internal emission in high-quality GaAs has been experimentally tested to be $99.7 \%$ [6]. GaAs is a unique material in that it both absorbs and radiates well, enabling the high voltages required to reach $>30 \%$ efficiency. 
The idea that increasing light emission improves open-circuit voltage seems paradoxical, as it is tempting to equate light emission with loss. Basic thermodynamics dictates that materials that absorb sunlight must also emit in proportion to their absorptivity. Thus, electron-hole recombination producing external luminescent emission is a necessity in solar cells. At open circuit, external photon emission is part of a necessary and unavoidable equilibration process, which does not represent loss at all.

At open circuit, an ideal solar cell would, in fact, radiate out of the solar cell a photon for every photon that was absorbed. Any additional nonradiative recombination, or photon loss, would indeed waste photons and electrons. Thus, the external luminescence efficiency is a gauge or an indicator of whether the additional loss mechanisms are present. In the case of no additional loss mechanisms, we can look forward to $100 \%$ external luminescence and maximum open-circuit voltage $V_{\mathrm{OC}}$. At the power-optimized solar cell operating bias point [15], the voltage is slightly reduced, and $98 \%$ of the open-circuit photons are drawn out of the cell as real current. Good external extraction comes at no penalty in current at the operating bias point.

On thermodynamic grounds, Ross [7] had already proposed that the open-circuit voltage would be penalized by poor external luminescence efficiency $\eta_{\text {ext }}$ as

$$
q V_{\mathrm{OC}}=q V_{\mathrm{OC}-\text { Ideal }}-k T\left|\ln \eta_{\text {ext }}\right| .
$$

This can be derived as follows: Under ideal open-circuit, quasiequilibrium conditions, the solar pump rate equals the external radiative rate: $R_{\text {ext }}=P_{\text {pump }}$. If the radiative rate is diminished by a poor external luminescence efficiency $\eta_{\text {ext }}$, the remaining photons must have been wasted in nonradiative recombination or parasitic optical absorption. The effective solar pump is then reduced to $P_{\text {pump }} \times \eta_{\text {ext }}$. The quasi-equilibrium condition is then $R_{\text {ext }}=P_{\text {pump }} \times \eta_{\text {ext }}$ at open circuit. Since the radiative rate $R_{\text {ext }}$ depends on the carrier density $n p$ product, which is proportional to $\exp \left\{q V_{\mathrm{OC}} / k T\right\}$, then the poor extraction $\eta_{\text {ext }}$ penalizes $V_{\mathrm{OC}}$, just as indicated in (1).

Another way of looking at this is to notice the shorter carrier lifetime in the presence of the additional nonradiative recombination. We start with a definition $\eta_{\text {ext }} \equiv R_{\text {ext }} /\left(R_{\text {ext }}+R_{\mathrm{nr}}\right)$, where $R_{\mathrm{nr}}$ is the internal photon and carrier nonradiative loss rate per unit area. Simple algebraic manipulation shows that the total loss rate $\left(R_{\text {ext }}+R_{\mathrm{nr}}\right)=R_{\text {ext }} / \eta_{\text {ext }}$. Thus, a poor $\eta_{\text {ext }}<1$ increases the total loss rate in inverse proportion, and the shorter lifetime limits the buildup of carrier density at open circuit. Then, carrier density is connected to $\exp \left\{q V_{\mathrm{OC}} / k T\right\}$ as before.

It is important to emphasize that light emission should occur opposite to the direction of the incident photons. A maximally concentrating solar cell would emit photons only directly back to the sun, thus achieving even higher voltages [16], [17]. However, concentrators miss the substantial fraction of diffuse sunlight; therefore, we focus instead on nonconcentrating solar cells. Such cells absorb both direct and diffuse sunlight from all incident angles. The unavoidable balancing emission is that of luminescent photons exiting through the front. Consequently, light emission only from the front surface should be maximized. Having a good mirror on the rear surface greatly improves the front-surface luminescent photon extraction and, therefore, the voltage.

\section{TheOreticAl EFFICIENCY Limits OF GAAs SOlar CELls}

The SQ limit includes a major role for external luminescence from solar cells. Accordingly, internal luminescence followed by light extraction plays a direct role in determining theoretical efficiency. To understand these physical effects, a specific material system must be analyzed, replacing the hypothetical step function absorber stipulated by SQ.

GaAs is a good material example, where external luminescence extraction plays an important role in determining the fundamental efficiency prospects. The quasi-equilibrium approach developed by SQ [1] is the most rigorous method for calculating such efficiency limits. Properly adapted, it can account for the precise incoming solar radiation spectrum, the real material absorption spectrum, the internal luminescence efficiency, as well as the external extraction efficiency and light trapping [12]. Calculations including such effects for Silicon solar cells were completed more than 25 years ago [12], [18]. Surprisingly, a calculation with the same sophistication has yet to be completed for GaAs solar cells.

Previous GaAs calculations have approximated the solar spectrum to be a blackbody at $6000 \mathrm{~K}$ and/or the absorption coefficient to be a step function [1], [19], [20]. The efficiency limits calculated with these assumptions are all less than or equal to $31 \%$.

In this paper, we calculate that the theoretical maximum efficiency of a GaAs solar cell, using [2] the 1-sun AM1.5G solar spectrum, and the proper absorption curve of GaAs, is in fact $33.5 \%$. Allowing for practical limitations, it should be possible to manufacture flat-plate single-junction GaAs solar cells with efficiencies above $30 \%$ in the near future. As we have already shown, realizing such efficiencies will require optical design such that the solar cell achieves optimal light extraction at open circuit.

Consider a solar cell in the dark surrounded by a thermal bath at room temperature $T$. The surrounding environment radiates at $T$ according to the tail $(E \gg k T)$ of the blackbody formula:

$$
b(E)=\frac{2 n_{r}^{2} E^{2}}{h^{3} c^{2}} \exp \left(\frac{-E}{k T}\right)
$$

where $b$ is given in photons per unit area, per unit time, per unit energy, per steradian. $E$ is the photon energy, $n_{r}$ is the ambient refractive index, $c$ is the speed of light, and $h$ is Planck's constant. As Lambertian-distributed photons enter the solar cell's surface at polar angle $\theta$, with energy $E$, the probability they will be absorbed is written as the dimensionless absorbance $a(E, \theta)$. The flux per unit solid angle of absorbed photons is, therefore, $a(E, \theta) b(E)$. In thermal equilibrium, there must be an emitted photon for every absorbed photon; the flux of emitted photons per unit solid angle is then also $a(E, \theta) b(E)$.

When the cell is irradiated by the sun, the system will no longer be in thermal equilibrium. There will be a chemical potential separation $\mu$ between electron and hole quasi-Fermi levels. The emission spectrum, which depends on electrons and 

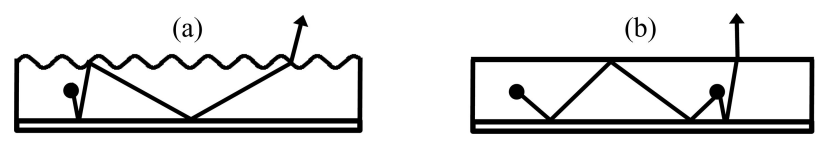

(c)

Fig. 3. Solar performance from three geometries. (a) Randomly textured front surface with a perfectly reflecting mirror on the rear. (b) Planar front surface with a perfectly reflecting mirror on the rear surface. (c) Planar front surface with an absorbing mirror on the rear surface.

holes coming together, will be multiplied by the normalized $n p$ product, i.e., $\left(n p / n_{i}^{2}\right)$, where $n, p$, and $n_{i}$ are the excited electron and hole concentration, and the intrinsic carrier concentration, respectively. The law of mass action is $n p=n_{i}^{2} \exp \{\mu / k T\}$ for the excited semiconductor in quasi-equilibrium, where $\mu$ is the internal chemical potential created by the sunlight. Then, the total photon emission rate is

$$
R_{\text {ext }}=\exp \left\{\frac{\mu}{k T}\right\} \iint a(E, \theta) b(E) d E \cos \theta d \Omega
$$

for external solid angle $\Omega$ and polar angle $\theta$. Equation (3) is normalized to the flat-plate area of the solar cell, meaning that the emission rate $R_{\text {ext }}$ is the emissive flux from only the front surface of the solar cell. We consider only nonconcentrating solar cells, meaning the solid angle integral is taken over the full hemisphere. There will, generally, be a much larger photon flux inside the cell, but most of the photons undergo total internal reflection upon reaching the semiconductor-air interface. If the rear surface is open to the air, i.e., there is no mirror, then the rear surface emission rate will equal the front-surface emission rate. As already discussed, restricting the luminescent emission to the front surface of the solar cell improves voltage, whereas a faulty rear mirror increases the avoidable losses, significantly reducing the voltage.

To find the open-circuit voltage, we now equate the carrier recombination and generation rates. Carriers are generated by the incident solar radiation $S(E)$ according to the formula

$$
P_{\text {pump }}=\iint a(E, \theta) S(E) d E \cos \theta d \Omega .
$$

Equating the generation and recombination rates, and recognizing that the open-circuit voltage equals the quasi-Fermi level separation $\left(q V_{\mathrm{OC}}=\mu\right)$, the resulting open-circuit voltage is

$$
\begin{aligned}
V_{\mathrm{OC}}= & \frac{k T}{q} \ln \left\{\frac{\iint a(E, \theta) S(E) d E \cos \theta d \Omega}{\iint a(E, \theta) b(E) d E \cos \theta d \Omega}\right\} \\
& -\frac{k T}{q}\left|\ln \left\{\eta_{\text {ext }}\right\}\right|
\end{aligned}
$$

which is an expanded version of (1). Since $\eta_{\text {ext }}$ is $\leq 1$, the second term in (5) represents a loss of voltage due to poor light extraction.

To explore the physics of light extraction, we consider GaAs solar cells with three possible geometries, as shown in Fig. 3. The first geometry [see Fig. 3(a)] is the most ideal, with a randomly textured front surface and a perfectly reflecting mirror on the rear surface. The surface texturing enhances absorption and improves light extraction, while the mirror ensures that the photons exit from the front surface and not the rear. The second geometry [see Fig. 3(b)] uses a planar front surface while retaining the perfectly reflecting mirror. Finally, the third geometry

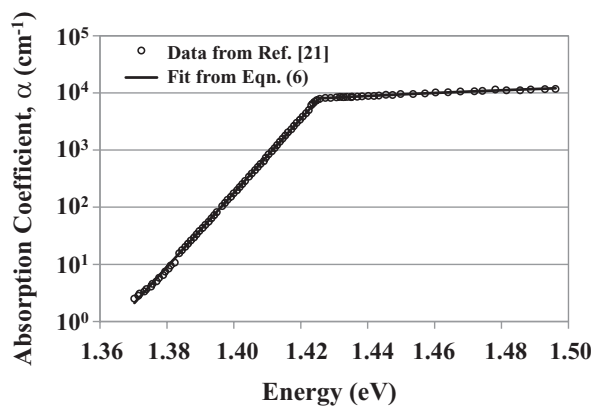

Fig. 4. GaAs absorption coefficient as a function of energy. GaAs has a direct bandgap at $E_{g}=1.42 \mathrm{eV}$, with an "Urbach tail" that falls off exponentially for lower energies.

[see Fig. 3(c)] has a planar front surface and an absorbing rear mirror, which captures most of the internally emitted photons before they can exit the front surface. We will show that this configuration achieves almost the same short-circuit current as the others, but suffers greatly in voltage and, consequently, efficiency. Thus, the optical design affects the voltage more than it does the current. Note that the geometry in Fig. 3(c) is equivalent to the common situation in which the active layer is epitaxially grown on top of an electrically passive substrate, which absorbs without reemission.

GaAs has a 1.4-eV bandgap that is ideally suited for solar cells. It is a direct-bandgap material, with an absorption coefficient of $8000 \mathrm{~cm}^{-1}$ near its band edge. By contrast, the absorption coefficient of $\mathrm{Si}$ is $\sim 10^{4}$ times weaker at its indirect band edge. Fig. 4 shows a semi-log plot of the absorption coefficient as a function of energy; the circles represent experimental data from [21], while the solid line represents a fit to the data using the piecewise continuous function:

$$
\alpha= \begin{cases}\alpha_{0} \exp \left(\frac{E-E_{g}}{E_{0}}\right) & E \leq E_{g}, \\ \alpha_{0}\left(1+\frac{E-E_{g}}{E^{\prime}}\right) & E>E_{g},\end{cases}
$$

where $\alpha_{o}=8000 / \mathrm{cm}$, the Urbach energy is $E_{o}=6.7 \mathrm{meV}$, and $E^{\prime}=140 \mathrm{meV}$. The exponential dependence of the absorption coefficient below the bandgap is characteristic of the "Urbach tail" [22].

Efficient external emission can be separated into two steps. First, the semiconductor should have a substantially higher probability of recombining radiatively, rather than nonradiatively. We define the internal luminescence yield $\eta_{\text {int }}$, similarly to the external luminescence yield, as the probability of radiative recombination versus nonradiative recombination, i.e., $\eta_{\text {int }} \equiv$ $R_{\text {int }} /\left(R_{\text {int }}+R_{\mathrm{nr}}\right)$, where $R_{\text {int }}$ and $R_{\text {nr }}$ are the radiative and nonradiative recombination rates per unit volume, respectively. The 
internal luminescence yield is a measure of intrinsic material quality. The second factor for efficient emission is proper optical design to ensure that the internally radiated photons eventually make their way out to external surface of the cell. Maximizing both factors is crucial for high open-circuit and operating point voltages.

We now derive the external luminescence yield for the three different geometries. At open circuit, $P_{\text {pump }}$ and the recombination rates, i.e., $\left(R_{\text {ext }}+R_{\mathrm{nr}}\right)$, are equal, and this allowed the derivation of (5) for a general open-circuit voltage. In operation, however, current will be drawn from the solar cell and the two rates will not be equal. The current will be the difference between pump and recombination terms:

$$
\begin{aligned}
J= & q\left(P_{\text {pump }}-R_{\text {ext }}-R_{\mathrm{nr}}\right)=\int_{0}^{\infty} a(E) S(E) d E \\
& -\frac{1}{\eta_{\text {ext }}} q \pi e^{q V / k T} \int_{0}^{\infty} a(E) b(E) d E
\end{aligned}
$$

where the external luminescence from the cell is a Lambertian that integrates to $\pi$ steradians, and the absorption $a(E, \theta)$ has been assumed independent of polar angle $\theta$, which is clearly valid for the randomly textured surface. It is independent of incident angle for a planar front surface because the large refractive index of GaAs refracts the incident light very close to perpendicular inside the solar cell.

\section{A. Randomly Textured Surface}

Randomly texturing the front surface of the solar cell [see Fig. 3(a)] represents an ideal method for coupling incident light to the full internal optical phase space of the cell. The absorption of a textured cell has been derived in [23]:

$$
a(E)=\frac{4 n^{2} \alpha L}{4 n^{2} \alpha L+1} .
$$

Although only strictly valid in the weakly absorbing limit, the absorptivity is close enough to 1 for large $\alpha L$ that (8) can be used for all energies.

To derive the external luminescence yield, all of the recombination mechanisms must be identified. We have assumed a perfectly reflecting rear mirror; therefore, the net radiative recombination is the emission from the front surface, which is given by (3). The only fundamental nonradiative loss mechanism in GaAs is Auger recombination. The Auger recombination rate per unit area is $C L n_{i}^{3} \exp \{3 q V / 2 k T\}$, where $L$ is the thickness of the cell, $C=7 \times 10^{-30} \mathrm{~cm}^{6} \cdot \mathrm{s}^{-1}$ is the Auger coefficient [24], and intrinsic doping is assumed to minimize the Auger recombination. The external luminescence yield can then be written as

$$
\eta_{\text {ext }}(V)=\frac{\pi e^{q V / k T} \int_{0}^{\infty} a(E) b(E) d \mathrm{E}}{\pi e^{q V / k T} \int_{0}^{\infty} a(E) b(E) d \mathrm{E}+C L n_{i}^{3} e^{3 q V / 2 k T}} .
$$

\section{B. Planar Front Surface With Perfectly Reflecting Mirror}

A second interesting configuration to consider is that of Fig. 3(b), which has a planar front surface and a perfectly reflecting rear mirror. Comparison with the first configuration allows

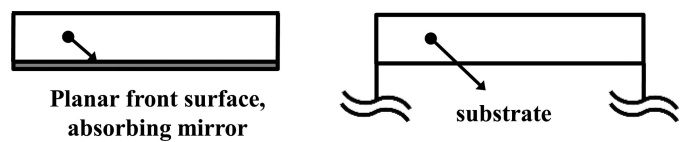

Fig. 5. Optically equivalent configurations, assuming the substrate does not reflect, nor reradiate, absorbed photons. In both cases, almost all of the internally emitted light will be lost out of the rear surface of the solar cell.

for explicit determination of the improvement introduced by random surface texturing. Not surprisingly, surface texturing only helps for very thin cells.

The absorptivity of the planar cell is well known

$$
a(E)=1-e^{-2 \alpha L}
$$

where the optical path length is doubled because of the rear mirror. Using this absorptivity formula, (3) still represents the external emission rate. As a consequence, the external luminescence yield follows the same formula, i.e., (9), albeit with a different absorptivity $a(E)$ for the planar front surface versus the textured solar cell.

\section{Planar Front Surface With Absorbing Mirror}

We have emphasized the importance of light extraction at open circuit to achieve a high voltage. To demonstrate the effects of poor optical design on efficiency, we also consider the geometry in Fig. 3(c). No extra recombination mechanism has been introduced, but the rear mirror now absorbs light rather than reflecting it internally. (Equivalently, it transmits light into a nonradiating, optically lossy, substrate.)

One could explicitly calculate the probability of internally emitted light escaping, tracking the photons to calculate the external luminescent yield. However, a simpler approach is to realize that the geometry with an absorbing rear mirror is equivalent to a setup with an absorbing nonluminescent substrate supporting the active material, as depicted in Fig. 5. Viewed either way, the absorptivity is $a(E)=1-e^{-\alpha L}$, where the light now has the opportunity for only one pass through the semiconductor to become absorbed.

To calculate the external luminescence yield, one can use the rate balancing method described earlier. The recombination terms for emission out of the front surface and Auger processes are still present. Now, there is also a term for emission out of the rear surface. By the same reasoning as for front-surface emission, the emission out of the rear surface balances the thermal radiation coming from below: $a^{\prime}\left(E, \theta^{\prime}\right) \times b^{\prime}(E) \times \exp \{q V / k T\}$, which includes a further boost by the quasi-equilibrium factor $\exp \{q V / k T\}$. At the rear surface, the density of states of the internal blackbody radiation $b^{\prime}(E) \equiv n_{r}^{2} b(E)$ is increased by $n_{r}^{2}$, where $n_{r}$ is the refractive index of the semiconductor. The rear absorption $a^{\prime}(E, \theta)$ is also modified as shown in the following equation for the total number of incident photons absorbed per unit area:

$$
2 \pi n_{r}^{2} \int_{0}^{\infty} b(E) \int_{0}^{\pi / 2}\left(1-e^{-f\left(\theta^{\prime}\right) \alpha L / \cos \theta^{\prime}}\right) \cos \theta^{\prime} \sin \theta^{\prime} d \theta^{\prime}
$$




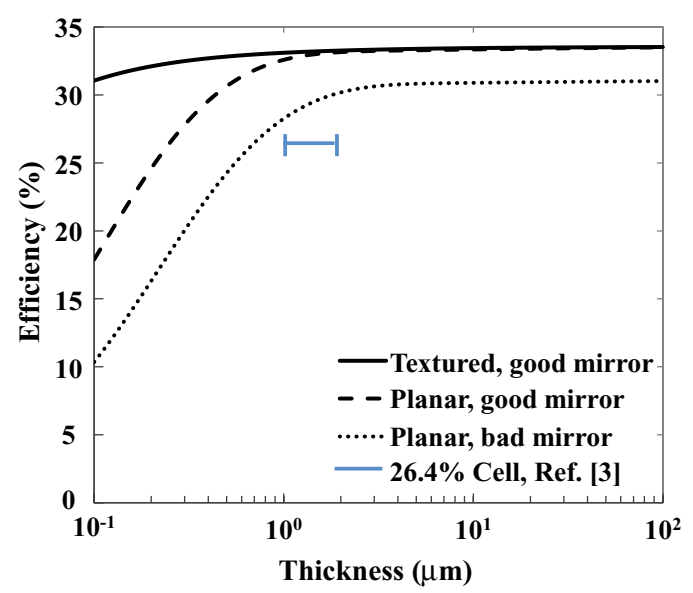

Fig. 6. GaAs solar cell efficiency as a function of thickness. Random surface texturing does not increase the limiting efficiency of $33.5 \%$, although it enables the high efficiencies, even for cell thicknesses less than $1 \mu \mathrm{m}$. Having an absorbing mirror on the rear surface incurs a voltage penalty and reduces the theoretical limiting efficiency to $31.1 \%$. There is still a sizeable gap between the $26.4 \%$ cell and the theoretical limit. The cell thickness was not specified in [3] and has been estimated as $1-2 \mu \mathrm{m}$.

where the $2 \pi$ prefactor arises from the azimuthal integral, and $f\left(\theta^{\prime}\right)$ equals one (two) for photons inside (outside) the escape cone, accounting for the different path lengths traveled by internal photons at angles greater or less than the critical angle $\theta_{c}$, which is defined by the escape cone at the top surface. The internal path length by oblique rays is increased by the factor $1 / \cos \theta^{\prime}$. A similar expression for the rear absorption is found in [20]. The external luminescence yield is now the ratio of the emission out of the front surface to the sum of the emission out of either surface plus Auger recombination, as in (12), shown at the bottom of the page, which is an explicit function of the quasi-Fermi level separation $q V$.

Given the absorptivity and external luminescence yield of each geometry, calculation of the solar cell's $I-V$ curve and power conversion efficiency is straightforward using (7). The power output of the cell $P$ is simply the current multiplied by the voltage. The operating point (i.e., the point of maximum efficiency) is the point at which $d P / d V=0$. Substituting the absorption coefficient data and solar spectrum values into (7), it is simple to numerically evaluate the bias point where the derivative of the output power equals zero.

Fig. 6 shows a plot of the solar cell efficiencies as a function of thickness for the three solar cell configurations considered.

Also included is a horizontal line representing the best GaAs solar cell fabricated up to 2010, which had an efficiency of $26.4 \%$ [3]. The maximum theoretical efficiency is $33.5 \%$, more than $7 \%$ larger in absolute efficiency. An efficiency of 33.5\% is theoretically achievable for both planar and textured front surfaces, provided there is a mirrored rear surface.
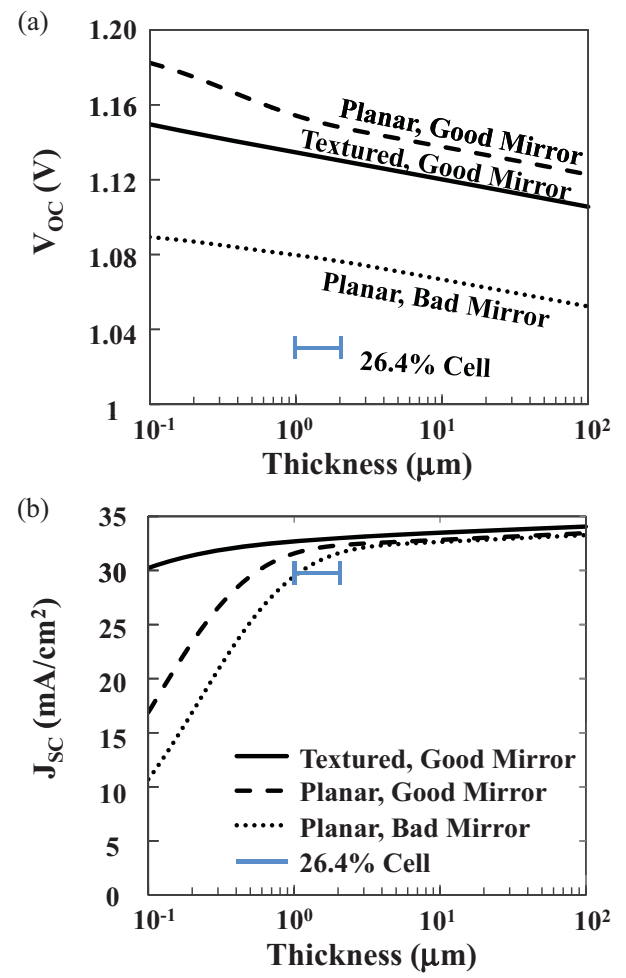

Fig. 7. (a) Open-circuit voltage $V_{\mathrm{OC}}$ and (b) short-circuit current $J_{\mathrm{SC}}$, as a function of thickness for each of the solar cell configurations considered. The planar cell with an absorbing mirror reaches almost the same short-circuit current as the other two configurations, but it suffers a severe voltage penalty due to poor light extraction and, therefore, lost photon recycling. There is considerable opportunity to increase $V_{\mathrm{OC}}$ over the previous record $26.4 \%$ cell. (The textured cell/good mirror has a lower voltage than a planar cell/good mirror owing to the effective bandgap shift observed in Fig. 10. This slight voltage drop is not due to poor $\eta_{\text {ext. }}$ )

Although surface texturing does not increase the maximum efficiency, it does help maintain an efficiency greater than $30 \%$ even for solar cells that are only a few hundred nanometers thick. The cell with a planar surface and bad mirror on its rear surface reaches an efficiency limit of only $31.1 \%$, exhibiting the penalty associated with poor light extraction. To understand more clearly the differences that arise in each of the three configurations, the short-circuit currents and open-circuit voltages of each are plotted in Fig. 7.

Table I and Fig. 7 display the differences in performance between a planar solar cell with a perfect mirror and one with an absorbing mirror. Although the short-circuit currents are almost identical for both mirror qualities at thicknesses greater than 2-3 $\mu \mathrm{m}$, the voltage differences are drastic. Instead of reflecting photons back into the cell where they can be reabsorbed, the absorbing mirror constantly removes photons from the system. The photon recycling process attendant to a high external luminescence yield is almost halted when the mirror is highly absorbing.

$$
\eta_{\operatorname{ext}}(V)=\frac{\pi e^{q V / k T} \int_{0}^{\infty} a(E) b(E) d \mathrm{E}}{\pi e^{q V / k T} \int_{0}^{\infty} b(E)\left[a(E)+2 n_{r}^{2} \int_{0}^{\pi / 2}\left(1-e^{-f\left(\theta^{\prime}\right) \alpha L / \cos \theta^{\prime}}\right) \sin \theta^{\prime} \cos \theta^{\prime} d \theta^{\prime}\right] d E+C L n_{i}^{3} e^{3 q V / 2 k T}}
$$


TABLE I

$V_{\mathrm{OC}}, J_{\mathrm{SC}}$, AND EFficiency VAlues For Three Possible GeOMETRIeS AND ReleVant Cell ThickNesses

\begin{tabular}{|c|c|c|c|c|c|c|c|c|c|}
\hline \multirow[b]{2}{*}{ Thickness } & \multicolumn{3}{|c|}{ Textured, good mirror } & \multicolumn{3}{|c|}{ Untextured, good mirror } & \multicolumn{3}{|c|}{ Untextured, bad mirror } \\
\hline & $500 \mathrm{~nm}$ & $1 \mu \mathrm{m}$ & $10 \mu \mathrm{m}$ & $500 \mathrm{~nm}$ & $1 \mu \mathrm{m}$ & $10 \mu \mathrm{m}$ & $500 \mathrm{~nm}$ & $1 \mu \mathrm{m}$ & $10 \mu \mathrm{m}$ \\
\hline Voc (volts) & 1.14 & 1.13 & 1.12 & 1.16 & 1.15 & 1.14 & 1.08 & 1.08 & 1.07 \\
\hline $\mathrm{Jsc}\left(\mathrm{mA} / \mathrm{cm}^{2}\right)$ & 32.3 & 32.7 & 33.5 & 29.5 & 31.6 & 32.8 & 25.2 & 29.5 & 32.6 \\
\hline Fill Factor & 0.89 & 0.89 & 0.89 & 0.89 & 0.89 & 0.89 & 0.89 & 0.89 & 0.89 \\
\hline efficiency $\%$ & 32.8 & 33.1 & 33.4 & 30.6 & 32.6 & 33.3 & 24.3 & 28.3 & 30.9 \\
\hline
\end{tabular}

A good rear mirror is crucial to a high open-circuit voltage and, consequently, to efficiencies above $30 \%$.
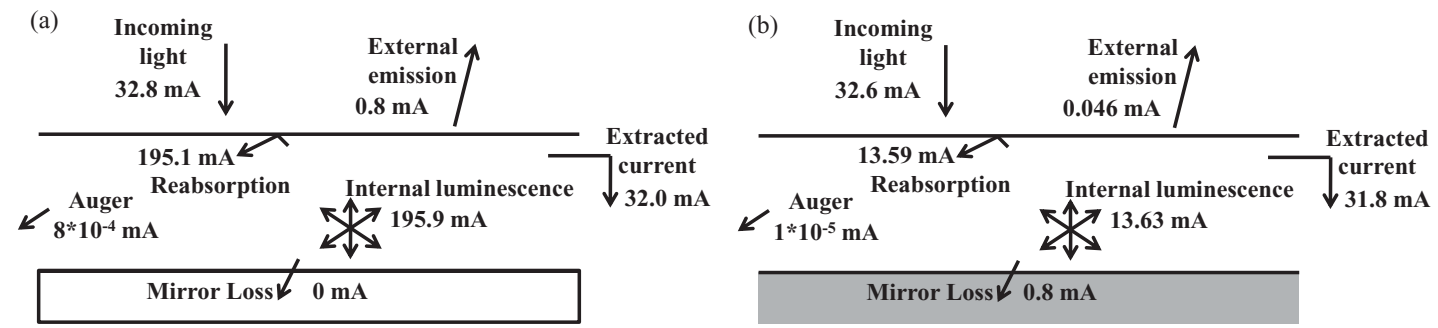

Fig. 8. Diagram of currents at the operating point for a planar $10-\mu \mathrm{m}$-thick $1-\mathrm{cm}^{2}$ solar cell (a) with a perfectly reflecting mirror and (b) with an absorbing mirror. The two solar cells produce almost the same amount of current, but the cell with a perfectly reflecting mirror achieves a higher voltage. The internal luminescence and reabsorption demonstrate the impact of front-surface emission on carrier densities. In GaAs, Auger recombination is negligible.

Fig. 8 presents such intuition visually, displaying the internal and external currents of a $10-\mu$ m-thick GaAs solar cell at its maximum power point, for $0 \%$ and $100 \%$ reflectivity. In both cases, the cells absorb very well, and the short-circuit currents are almost identical. The extracted currents, too, are almost identical. In each case, $0.8 \mathrm{~mA}$ of current is "lost" (i.e., the difference between short-circuit and operating currents), but in the case of the good mirror, the current is lost to front-surface emission. There is a strong buildup of photon density when the only loss is emission through the front surface, allowing much higher internal luminescence and carrier density. A higher operating voltage results.

From Fig. 6, it is clear that surface texturing is not helpful in GaAs, except to increase current in the very thinnest solar cells. In most solar cells, such as Silicon cells, surface texturing provides a mechanism for exploiting the full internal optical phase space. The incident sunlight is refracted into a very small solid angle within the cell, and without randomizing reflections, photons would never couple to other internal modes.

GaAs is such an efficient radiator that can provide the angular randomization by photon recycling. After absorbing a photon, the photon will likely be reemitted, and the reemission is equally probable into all internal modes. Although most materials require surface roughness to efficiently extract light, the radiative efficiency of GaAs ensures light extraction based on photon recycling. Such photon dynamics are illustrated in Fig. 9.

In [20] and [25], the benefits of photon recycling toward efficiency have already been emphasized, but we believe that external luminescence yield is the more comprehensive parameter for boosting solar cell efficiency and voltage.
It seems surprising that the planar solar cell would have a higher voltage than the textured cell. This is due to a secondorder effect seen in Fig. 10. Textured cells experience high absorption, even below the bandgap, due to the longer optical path length provided. Texturing effectively reduces the bandgap slightly, as shown in Fig. 10, accounting for the lower open-circuit voltage but larger short-circuit current values seen in Fig. 7(b).

\section{DISCUSSION}

GaAs is an example of one of the very few material systems that can reach internal luminescence yields close to 1 ; a value of $99.7 \%$ has been experimentally confirmed [6]. However, it is the external luminescence yield that determines voltage, and that yield depends on both the quality of material, as well as the optical design. Absorbing contacts, or a faulty rear mirror, for example, will remove photons from the system that could otherwise be recycled. Additionally, an optically textured design [13] can provide the possibility for extraction of luminescent photons, before they could be lost.

Light trapping is normally thought of as a way to absorb more light and increase the current in a thinner cell. However, the concentration of carriers in a thinner cell also provides a voltage increase, i.e., $q \Delta V \sim k T \ln \left\{4 n^{2}\right\}$, which is an effect that was implicitly used when light trapping was first incorporated into the fundamental calculation [12] of Silicon efficiency. Thus, texturing improves the voltage in most solar cells. Nonetheless, one of the main results of this paper is that the voltage boost can come with or without surface texturing in GaAs. The reason is that efficient internal photon recycling provides the angular 

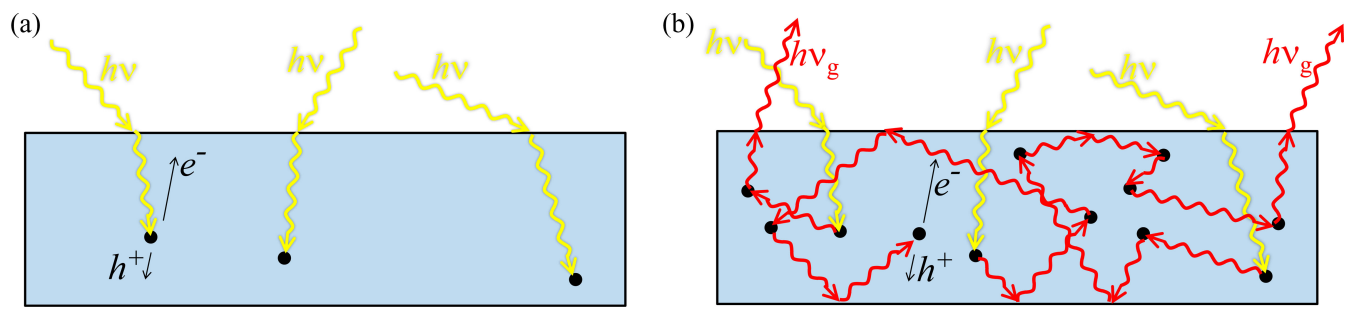

Fig. 9. Qualitative illustration of the different photon dynamics in plane-parallel solar cells with (a) low luminescence yield $\eta_{\text {ext }}$ and (b) high $\eta_{\text {ext }}$, respectively. In (a), the lack of photon extraction reduces carrier density and, thus, voltage. Conversely, in (b), the internal photons achieve full angular randomization, even without surface texturing, and the high external emission is indicative of high carrier density buildup.

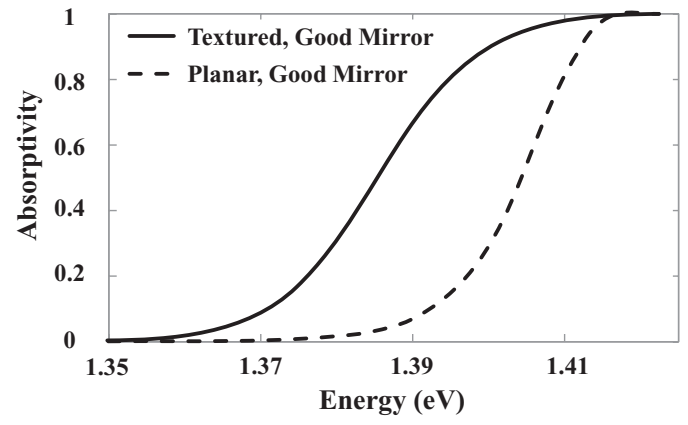

Fig. 10. Absorptivity of a 10- $\mu$ m-thick cell as a function of photon energy near the bandgap. A textured cell absorbs well even at $1.39 \mathrm{eV}$, effectively reducing the bandgap. This explains the lower voltages but higher currents of the textured cell, relative to the plane-parallel cell, in Fig. 7.

randomization necessary to concentrate the light, even in a plane-parallel GaAs cell. Thus, short-circuit current in GaAs can benefit from texturing, but GaAs voltage accrues the same benefit with, or even without, texturing.

The distinction between voltage boost by texturing and voltage boost by photon recycling was already made by Lush and Lundstrom [26], who predicted the higher voltages and the record efficiencies that have recently been [27] observed in thinfilm III-V solar cells. However, the overarching viewpoint in this paper is that voltage is determined by external luminescence efficiency. That viewpoint accounts in a single comprehensive manner for the benefits of nanotexturing, photon recycling, parasitic optical reflectivity, and imperfect luminescence, while being thermodynamically self-consistent.

In the case of perfect photon recycling, there is surprisingly little thickness dependence of $V_{\mathrm{OC}}$. This is to be contrasted with the textured case, where the voltage boost might require light concentration and carrier concentration within a thin cell. Under perfect photon recycling, photons are lost only at the surface, and the photon density and carrier density are maintained at the maximum value through the full depth. The solar cell can be permitted to become thick, with no penalty. In practice, a thick cell would carry a burden, and an optimum thickness would emerge.

\section{CONCLUSION}

We have shown how to include photon recycling and imperfect radiation properties into the quasi-equilibrium formulation of Shockley and Queisser. High voltages $V_{\mathrm{OC}}$ are achieved by maximizing the external luminescence yield of a system. Using the standard solar spectrum and the measured absorption curve of GaAs, we have shown that the theoretical efficiency limit of GaAs is $33.5 \%$, which is more than $4 \%$ higher than that of Silicon [28], and achieves its efficiency in a cell that is 100 times thinner.

Internally trapped radiation is necessary, but not sufficient, for the high external luminescence that allows a cell to reach voltages near the theoretical limits. The optical design must ensure that the only loss mechanism is photons exiting at the front surface. A slightly faulty mirror, or equivalently absorbing contacts or some other optical loss mechanism, sharply reduces the efficiency limit that can be achieved. To realize solar cells with efficiency greater than $30 \%$, the optical configuration will need to be very carefully designed.

The prior [3] 1-sun, single-junction efficiency record, i.e., $26.4 \%$, was set by GaAs cells that had $V_{\mathrm{OC}}=1.03 \mathrm{~V}$. Alta Devices has recently [27] made a big improvement in GaAs efficiency and open-circuit voltage, i.e., $28.3 \%$ and $\mathrm{V}_{\mathrm{OC}}=1.11 \mathrm{~V}$, respectively, showing in part the benefit of light extraction.

The SQ formulation is still the foundation of solar cell technology. However, the physics of light extraction and external luminescence yield are clearly relevant for high-performance cells and will prove important in the eventual determination of which solar cell technology wins out in the end. In the push for high-efficiency solar cells, a combination of high-quality GaAs and optimal optical design should enable single-junction flat-plate solar cells with greater than $30 \%$ efficiency.

\section{REFERENCES}

[1] W. Shockley and H. J. Queisser, "Detailed balance limit of efficiency of p-n junction solar cells," J. Appl. Phys., vol. 32, pp. 510-519, Mar. 1961.

[2] National Renewable Energy Laboratory. Reference Solar Spectral Irradiance: Air Mass 1.5, ASTM G-173-03. (2012). [Online]. Available at http://rredc.nrel.gov/solar/spectra/am1.5/

[3] M. A. Green, K. Emery, Y. Hishikawa, and W. Warta, "Solar cell efficiency tables (version 36)," Progr. Photovoltaic: Res. Appl., vol. 18, pp. 346-352, Jun. 2010.

[4] G. J. Bauhuis, P. Mulder, E. J. Haverkamp, J. C. C. M. Huijben, and J. J. Schermer, "26.1\% thin-film GaAs solar cell using epitaxial lift-off," Solar Energy Mater. Solar Cells, vol. 93, pp. 1488-1491, May 2009.

[5] M. A. Green, K. Emery, Y. Hishikawa, and W. Warta, "Solar cell efficiency tables (version 31)," Progr. Photovoltaic: Res. Appl., vol. 16, pp. 61-67, 2008.

[6] I. Schnitzer, E. Yablonovitch, C. Caneau, and T. J. Gmitter, "Ultrahigh spontaneous emission quantum efficiency, $99.7 \%$ internally and $72 \%$ externally, from $\mathrm{AlGaAs} / \mathrm{GaAs} / \mathrm{AlGaAs}$ double heterostructures," Appl. Phys. Lett., vol. 62, pp. 131-133, Jan. 1993. 
[7] R. T. Ross, "Some thermodynamics of photochemical systems," J. Chem. Phys., vol. 46, pp. 4590-4593, Jun. 1967.

[8] M. A. Green, "Radiative efficiency of state-of-the-art photovoltaic cells," Progr. Photovoltaic: Res. Appl., vol. 20, pp. 472-476, Jun. 2012.

[9] J. Zhao, A. Wang, M. A. Green, and F. Ferrazza, " $19.8 \%$ efficient 'honeycomb' textured multicrystalline and $24.4 \%$ monocrystalline silicon solar cells," Appl. Phys. Lett., vol. 73, pp. 1991-1993, Oct. 1998.

[10] T. Trupke, J. Zhao, A. Wang, R. Corkish, and M. A. Green, "Very efficient light emission from bulk crystalline silicon," Appl. Phys. Lett., vol. 82, pp. 2996-2998, May 2003.

[11] E. Yablonovitch and T. Gmitter, "Auger recombination in silicon at low carrier densities," Appl. Phys. Lett., vol. 49, pp. 587-589, Sep. 1986.

[12] T. Tiedje, E. Yablonovitch, G. D. Cody, and B. G. Brooks, "Limiting efficiency of Silicon solar cells," IEEE Trans. Electron Devices, vol. ED-31, no. 5, pp. 711-716, May 1984.

[13] E. Yablonovitch and G. D. Cody, "Intensity enhancement in textured optical sheets for solar cells," IEEE Trans. Electron Devices, vol. ED-29, no. 2, pp. 300-305, Feb. 1982.

[14] W. van Roosbroeck and W. Shockley, "Photon-radiative recombination of electrons and holes in Germanium," Phys. Rev., vol. 94, p. 1558, 1954.

[15] P. Wurfel, Physics of Solar Cells. New York: Wiley-VCH, 1995, p. 124.

[16] G. L. Araújo and A. Marti, "Absolute limiting efficiencies for photovoltaic energy conversion," Solar Energy Mater. Solar Cells, vol. 33, pp. 213240, Jun. 1994.

[17] L. C. Hirst and N. J. Ekins-Daukes, "Fundamental losses in solar cells," Progr. Photovoltaic: Res. Appl., vol. 19, pp. 286-293, Aug. 2010.

[18] P. Campbell and M. A. Green, "The limiting efficiency of Silicon solar cells under concentrated sunlight," IEEE Trans. Electron Devices, vol. ED-33, no. 2, pp. 234-239, Feb. 1986.

[19] J. L. Balenzategui and A. Marti, "The losses of efficiency in a solar cell step by step," in Proc. 14th Eur. Photovoltaic Solar Energy Conf., 1997, pp. 2374-2377.
[20] A. Marti, J. L. Balenzategui, and R. F. Reyna, "Photon recycling and Shockley's diode equation," J. Appl. Phys., vol. 82, pp. 4067-4075, Oct. 1997.

[21] M. D. Sturge, "Optical absorption of Gallium Arsenide between 0.6 and 2.75 eV," Phys. Rev., vol. 127, pp. 768-773, Aug. 1962.

[22] F. Urbach, "The long-wavelength edge of photographic sensitivity and of the electronic absorption of solids," Phys. Rev., vol. 92, pp. 1324-1324, 1953.

[23] E. Yablonovitch, "Statistical ray optics," J. Opt. Soc. Amer., vol. 72, pp. 899-907, Jul. 1982.

[24] U. Strauss, W. W. Ruhle, and K. Kohler, "Auger recombination in intrinsic GaAs,” Appl. Phys. Lett., vol. 62, pp. 55-57, Jan. 1993.

[25] D. C. Johnson, I. M. Ballard, K. W. J. Barnham, J. P. Connolly, M. Mazzer, A. Bessière, C. Calder, G. Hill, and J. S. Roberts, "Observation of photon recycling in strain-balanced quantum well solar cells," Appl. Phys. Lett., vol. 90, pp. 213505-1-213505-3, May 2007.

[26] G. Lush and M. Lundstrom, "Thin film approaches for high-efficiency III-V cells," Solar Cells, vol. 30, pp. 337-344, May 1991.

[27] M. A. Green, K. Emery, Y. Hishikawa, W. Warta, and E. D. Dunlop, "Solar cell efficiency tables (version 39)," Progr.Photovoltaic: Res. Appl., vol. 20, pp. 12-20, Jan. 2012.

[28] M. J. Kerr, A. Cuevas, and P. Campbell, "Limiting efficiency of crystalline Silicon solar cells due to Coulomb-enhanced Auger recombination," Progr. Photovoltaic: Res. Appl., vol. 11, pp. 97-104, Mar 2003.

Authors' photographs and biographies not available at the time of publication. 